\title{
Nano Dimensional (1-20nm) Silver Nanoparticles: Stem Extract of Capparis decidua (FORSK.) EDGEW Mediated Synthesis and its Characterization-A Lab to Land Approach
}

\author{
Jyoti Ahlawat and Anita R. Sehrawat* \\ Department of Botany, Maharishi Dayanand University Rohtak, Haryana-124001, India \\ *Corresponding author
}

\begin{tabular}{|c|c|}
\hline & A B S T R A C T \\
\hline & \multirow{10}{*}{$\begin{array}{l}\text { A renewable innate source aqueous Capparis decidua stem extract was used to investigate } \\
\text { an eco-friendly approach for the synthesis of silver nanoparticles (AgNPs) from silver } \\
\text { nitrate solution. Biosynthesized silver nanoparticles were characterized by UV-visible } \\
\text { Spectroscopy, Transmission Electron Microscopy (TEM) and Fourier Transform Infra-Red } \\
\text { Spectroscopy (FTIR). The UV-visible spectroscopy showed the maximum absorbance i.e. } \\
0.94 \text { at } 460 \mathrm{~nm} \text {. The stability of nanoparticles after four months depicted almost no shift in } \\
\text { the absorption intensity and the absorption maxima which indicated that the particle size is } \\
\text { same. The broadening of peak specified that the particles were polydispersed. TEM } \\
\text { analysis conformed the formation of crystalline silver nanoparticles of size range } 1.0-19 \\
\text { nm. Very small size range of silver nanoparticles makes it a good antimicrobial agent. } \\
\text { Functional groups like amines, amides, alkynes, alkenes, bromoalkanes etc. identified by } \\
\text { FTIR analysis investigated the responsive functional groups which stabilises the } \\
\text { synthesized silver nanoparticles. The absorbance bands in bioreduction are observed in the } \\
\text { region of } 400-4000 \mathrm{~cm}^{-1} \text { by FTIR. }\end{array}$} \\
\hline Keywords & \\
\hline $\begin{array}{l}\mathrm{Ca} \\
\mathrm{Sil}\end{array}$ & \\
\hline $\begin{array}{l}\text { Silve } \\
\text { TEM }\end{array}$ & \\
\hline Poydis: & \\
\hline & \\
\hline Atrtcl & \\
\hline $\begin{array}{l}\text { Accepted: } \\
17 \text { Septeml }\end{array}$ & \\
\hline & \\
\hline & \\
\hline
\end{tabular}

\section{Introduction}

Today nano metal particles especially silver have drawn attention of scientist because of their extensive application in the development of new technologies in the areas of electronics, material sciences and medicine at the nano scale. Silver nanoparticles are nanoparticles of silver which are in the range of 1 and $100 \mathrm{~nm}$ in size. Silver nanoparticles have unique properties which help in molecular diagnostics, in therapies, as well as in devices that are used in several medical procedures. The major methods used for silver nanoparticle synthesis are the physical and chemical methods. Considering the drawbacks of physio-chemical methods, cost- effective and energy efficient new alternative for AgNP synthesis using microorganisms (Sharma et al., 2009), plant extracts (Song et al., 2009) and natural polymers (Huang et al., 2004) as reducing and capping agents are emerging very fast. The association of nanotechnology and green chemistry will unfold the range of biologically and cytologically compatible metallic nanoparticles which is a strong benevolent to lab to land approach. In physical synthesised silver nanoparticles by laser ablation method the properties of particles synthesised depends upon the wavelength and duration of the laser, the ablation time duration and the effective 
liquid medium which may or may not contain the surfactant (Mafune et al.,2000; Kabashin et al.,2003). The chemical synthesis approach is inevitably associated with the use of hazardous chemicals such as reductants, stabilizers and organic solvents, or has special requirements for the employed techniques such as high energy radiation, microwave irradiation and inert gas condensation (Shin et al., 2004; Mittal et al., 2004). Global warming and climate change has persuaded a worldwide awareness to reduce the toxic and hazardous waste materials as a result of this the green synthesis has gained progress in the fields of science and industry (Ahmad et al., 2010). In recent years, the biological approaches using microorganisms and plant extracts have become valuable alternatives to chemical synthesis. The physical and chemical processes often involve high temperatures/pressure for the reaction and the use of hazardous chemicals due to which the biological methods of synthesising metal nanoparticles is gaining importance. Plant extracts are considered cost-effective, environment friendly and efficient alternative for the large-scale synthesis of nanoparticles. Here the present work represents a stupendous effort to develop the eco-friendly, nontoxic and cost-effective approaches for the preparation of AgNPs without the application of toxic chemicals and special equipments.

The use of plant and plant extract in nanoparticle synthesis is considered advantageous over microbial based system because it reduces the elaborate process of maintaining cell cultures. In comparison to microorganisms, the application of plant extracts for the synthesis of AgNPs is more advantageous in terms of resource availability, security, reaction rate and convenience, and feasibility of large scale synthesis (Mane Gavde et al., 2015, Jalaluddin et al., 2016). The particle size, growth can also be controlled by altering synthesis conditions like $\mathrm{pH}$, reductant concentration, temperature, mixing ratio of the reactants etc. The plant based synthesis can be carried out either extracellularly or intracellularly. Intracellular synthesis takes place inside the plant whereas the extracellular synthesis occurs in vitro. The findings reported that extracellular synthesis using plant extracts has been considered better as compared to intracellular (Makarov et al., 2014) because it abolish the extraction and purification methods. Green synthesis have been reported in Magnifera indica (Sandeep et al., 2017), Corynebacterium species (Gowramma et al., 2015).

Till date, lot of papers has been published in this area which describes the mechanism and role of active biomolecules in synthesis. These studies suggested that presence of phytochemicals in plant extracts are the key component in reduction and stabilization of silver ions (Jegadeeswaran et al., 2012) The phytochemicals which are responsible for reduction are terpenoids, flavonoids, ketones, aldehydes, amides, and carboxylic acids. The water soluble metabolites such as flavones, organic acids, and quinones are solely responsible for the bioreduction ions. Some researchers have reported that a keto-enol transition of anthraquinone is responsible for formation of AgNPs. It has been also observed that mesophytes contain three types of benzoquinones: cyperoquinone, dietchequinone, and remirin which might be responsible for reduction of ions and formation of AgNPs (Nafeesa khatoon et al., 2017).

Capparis decidua (Family, Capparidaceae) is a shrub or small tree commonly known as 'ker' is an important medicinal plant. It is a rich source of beta-carotene (Talili et al., 2009) and mineral elements (Ozcan et al., 2008). The leaves of $C$. decidua possess phenolic compounds with multiple biological activities (Ramezani et al., 2008). Aerial parts (flower, fruit, stem and seed) contain n- 
triacontane, n-triacontanol, n-pentacosane, 6(1-hydroxy-non-3-enyl) tetrahydropyran-2one, 2-carboxy-1-dimethylpyrrolidine, $\beta$ sitosterol, $\quad \beta$-carotene, Glucosinolates, Ascorbic acid, proteins, carbohydrates, Calcium, Potassium, Phosphorous, Zinc, Iron and Manganese (Rathee et al., 2010). The quantity of $\beta$-sitosterol in the stem is found to be $0.044 \%$ by TLC densitometric method using HPTLC (Rathee et al., 2010).

\section{Materials and Methods}

\section{Preparation of the stem extract}

The explant was collected from village Samargopalpur (Rohtak, Haryana) India in December 2014. Stem extract of Capparis decidua was selected for the biosynthesis of AgNPs because of its ease of availability and medicinal properties. For biosynthesis Fresh and healthy part of stem were collected locally and rinsed thoroughly with tap water followed by doubled distilled water to remove all dust and unwanted visible particles, after which they were dried at room temperature to remove the water from the surface of stem, then cut into small pieces. Next, $20 \mathrm{~g}$ of these finely incised stem parts crushed in $100 \mathrm{ml}$ distilled water and boiled at $80^{\circ} \mathrm{C}$ for $20 \mathrm{~min}$.

After cooling at room temperature, the mixture was centrifuged at 20,000 rpm for 30 min and filtered. The filtrates were then stored at $4-8{ }^{\circ} \mathrm{C}$ and used as reducing and stabilizing agents in the synthesis of AgNPs. The sterile conditions were maintained throughout the experiment (Medda et al., 2014).

\section{AgNPs synthesis}

Aqueous solution of $1 \mathrm{mM}$ silver nitrate $\left(\mathrm{AgNO}_{3}\right)$ was prepared in a $250 \mathrm{ml}$ Erlenmeyer flask and used for the synthesis of AgNPs. Briefly, $12 \mathrm{ml}$ of Capparis decidua stem extract was added into $88 \mathrm{ml}$ of aqueous solution of $1 \mathrm{mM}$ silver nitrate and incubated in the dark overnight at room temperature. The primary step to detect complete reduction of $\mathrm{AgNO} 3$ to $\mathrm{Ag}+$ ions was confirmed by the change in colour from colorless to colloidal brownish yellow. The colloidal mixture was then sealed and stored properly for future use. The formation of AgNPs was further confirmed by spectrophotometric analysis. The silver nitrate (AgNO3) was purchased from Sigma-Aldrich, India.

\section{$\mathrm{UV}-\mathrm{V}$ is spectra analysis}

Preliminary characterization of the AgNPs was carried out using UV-Visible spectroscopy. The reduction of silver ions to the nanoparticle form was monitored by measuring the UV-Visible spectra of solutions after diluting the sample with Millipore water 20 times. The spectra of AgNPs solution was monitored by a UV-Vis spectrophotometer SHIMADZU UV-Vis Spectrophotometer 2450 and a UV-Vis spectrograph of the colloidal solution of silver nanoparticles was recorded as a function of time by using quartz cuvette with water as reference and scanning the spectra between $200-800 \mathrm{~nm}$ at the resolution of $0.1 \mathrm{~nm}$.

\section{Fourier transformation infrared (FTIR) spectroscopy}

FTIR spectroscopy was used to investigate the functional groups in the stem extract and their possible involvement in the synthesis of AgNPs. The modified functional groups in the green synthesized nanoparticles were determined with various concentrations of AgNPs. Buffer subtracted transmission spectra were recorded in the wave number range of $375-4000 \mathrm{~cm}^{-1}$ using a ALPHA Platinum ATR (attenuated total reflectance) FTIR spectrometer (Bruker, Germany) from the Department of Genetics, M.D.U Rohtak. 
The binding properties of AgNPs investigated by FTIR analysis and the difference between the respective binding agents are verified.

\section{Transmission electron microscopy (TEM)}

TEM grids were prepared by sonicating the AgNPs sample solution for $20 \mathrm{~min}$ and placing a few drops on the 300 mesh carboncoated copper grid and dried for the complete evaporation of water under a lamp and operated at an accelerating voltage of $100 \mathrm{kV}$ using EM2000Fx-II, transmission electron microscope, which is a (<Hitachi H-7500>, resolution $0.204 \mathrm{~nm}$, lattice image: $0.36 \mathrm{~nm}$ point to point, magnification: $6,00,000 \mathrm{x}$ ) with AMT XR-16 16mp high resolution CCDcamera, facility was availed from Sophisticated Analysis Instrument Facility, Panjab University, Chandigarh to characterize the sample after usual alignment procedures.

\section{Results and Discussion}

Silver nanoparticles were synthesized successfully from the stem extract of Capparis decidua with aqueous silver nitrate solution. The presence of nanoparticles in the medium was confirmed by the change in color from colorless to brown or deep yellow shown in (Fig. 1). The color intensity increased with a period of incubation due to the reduction in silver nanoparticles and excitation of surface plasmon vibrations (S.Li et al., 2007) and provides a convenient spectroscopic proof of their formation $(\mathrm{N}$. Saifuddin et al., 2009).

\section{UV-Vis spectrophotometer analysis}

The silver nanoparticles were characterized by UV-Vis spectroscopy, one of the most widely used techniques for structural characterization of silver nanoparticles (Sun et al., 2001). Biological method of silver nanoparticles synthesis exhibit strong absorption of electromagnetic waves in the visible range due to their optical resonant property, called occurs due to its collective oscillation of conduction electrons, combined with the incident light (Kreibig and Vollmer, 1995). Figure 2 shows the UV-Vis absorption spectra at $460 \mathrm{~nm}$ with 0.94 absorbance. This peak is due to Surface Plasmon Resonance (SPR) property of silver nanoparticles. Nanoparticles have optical properties that are sensitive to size, shape, concentration, agglomeration state, and refractive index near the nanoparticle surface, which makes UV/Vis/ IR spectroscopy a valuable tool for identifying, characterizing, and studying these materials (Shakeel Ahmad et al., 2016) Nanoparticles made from certain metals, such as gold and silver, strongly interact with specific wavelengths of light and the unique optical properties of these materials is the foundation for the field of plasmonics. The prepared aqueous solution of AgNPs showed a strong absorption band between 410 and $460 \mathrm{~nm}$, which is a typical absorption band of spherical Ag nanoparticles due to their surface plasmon and widening of the peak indicated that the particles were polydispersed (Fig. 2) Similar results were reported in Cassia italica (Sermakkani et al., 2012; Mulvney 1996; Shibila ans Johnson, 2015; Sai Lakshmi et al., 2015).

\section{TEM analysis of silver nanoparticles}

The well-known technique for imaging solid materials at atomic resolution is TEM. The technique was employed to visualize the size and shape of $\mathrm{Ag}$ nanoparticles. From our results, it is observed that most of the $\mathrm{Ag}$ nanoparticles were spherical in shape. Figure 3 clearly shows the TEM image of individual silver nanoparticles with the particle size estimated as in between the range of 1- 20 nm. The stem extract synthesized nanoparticles with spherical shape has also been reported in Cinnamon zeylanicum with 
size range of 31-40nm (Joyita Banerjee and Narendhirakannan, 2011), Shorea tumbuggaia and Boswellia ovalifoliolata (Harekrishna Bar et al., 2009). In the present work as per Transmission Electron Microscopy results the nanoparticles were found not in direct contact and even no aggregates were seen which is an indication of the stabilization of the nanoparticles by a capping agent (due to presence of proteins and secondary metabolites in the stem extract). Proteins have stronger ability to bind metal indicating that the proteins could be a covering layer around the AgNPs preventing agglomeration, hence, behaves as a capping agent for AgNPs (Sermakkani et al., 2012). The smaller range of size of silver nanoparticles of Capparis decidua makes it a better microcidal.

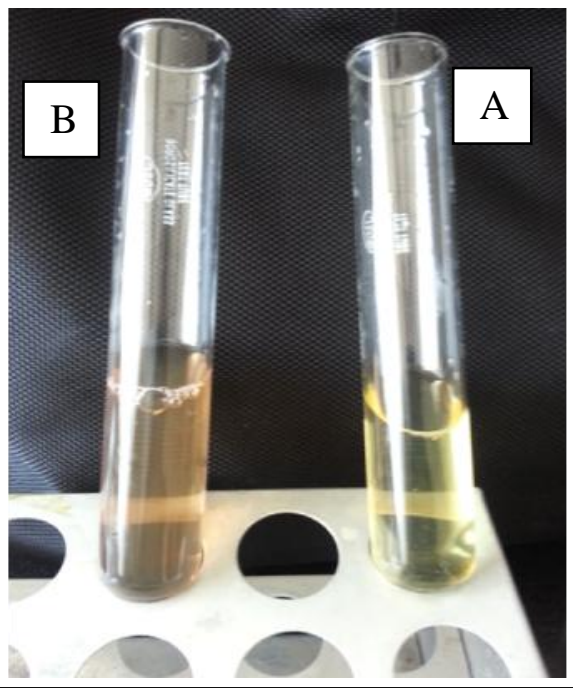

Fig.1 Capparis decidua (a) stem extract (b) silver nanoparticle synthesized

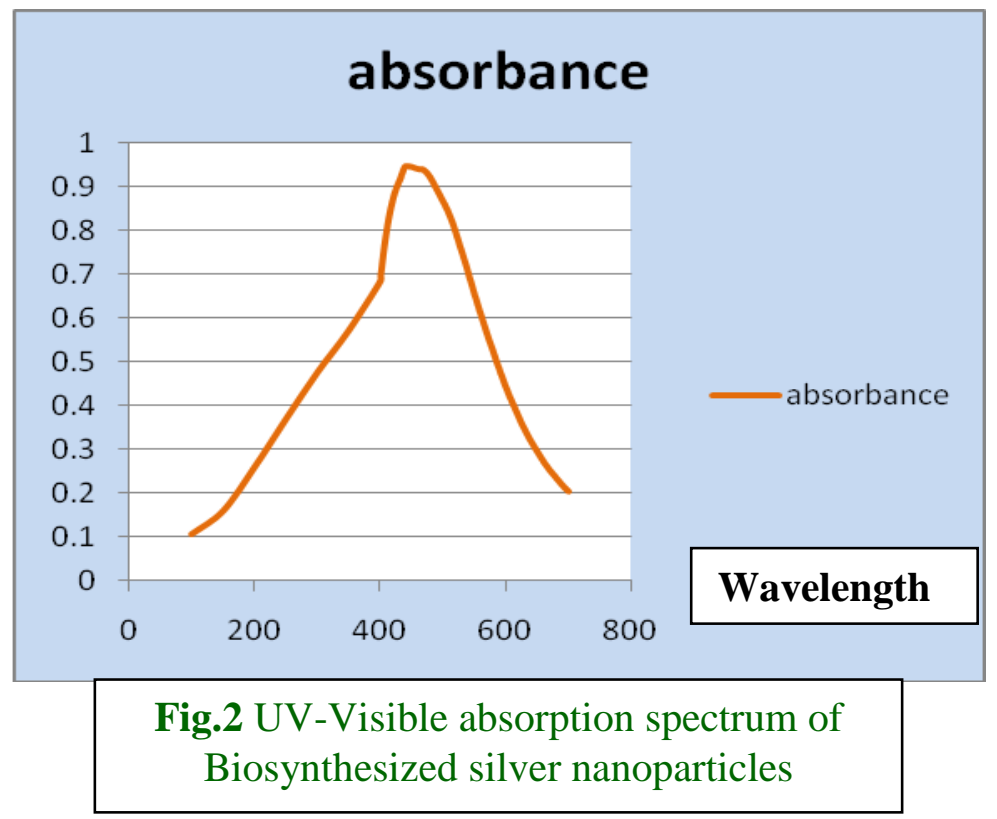



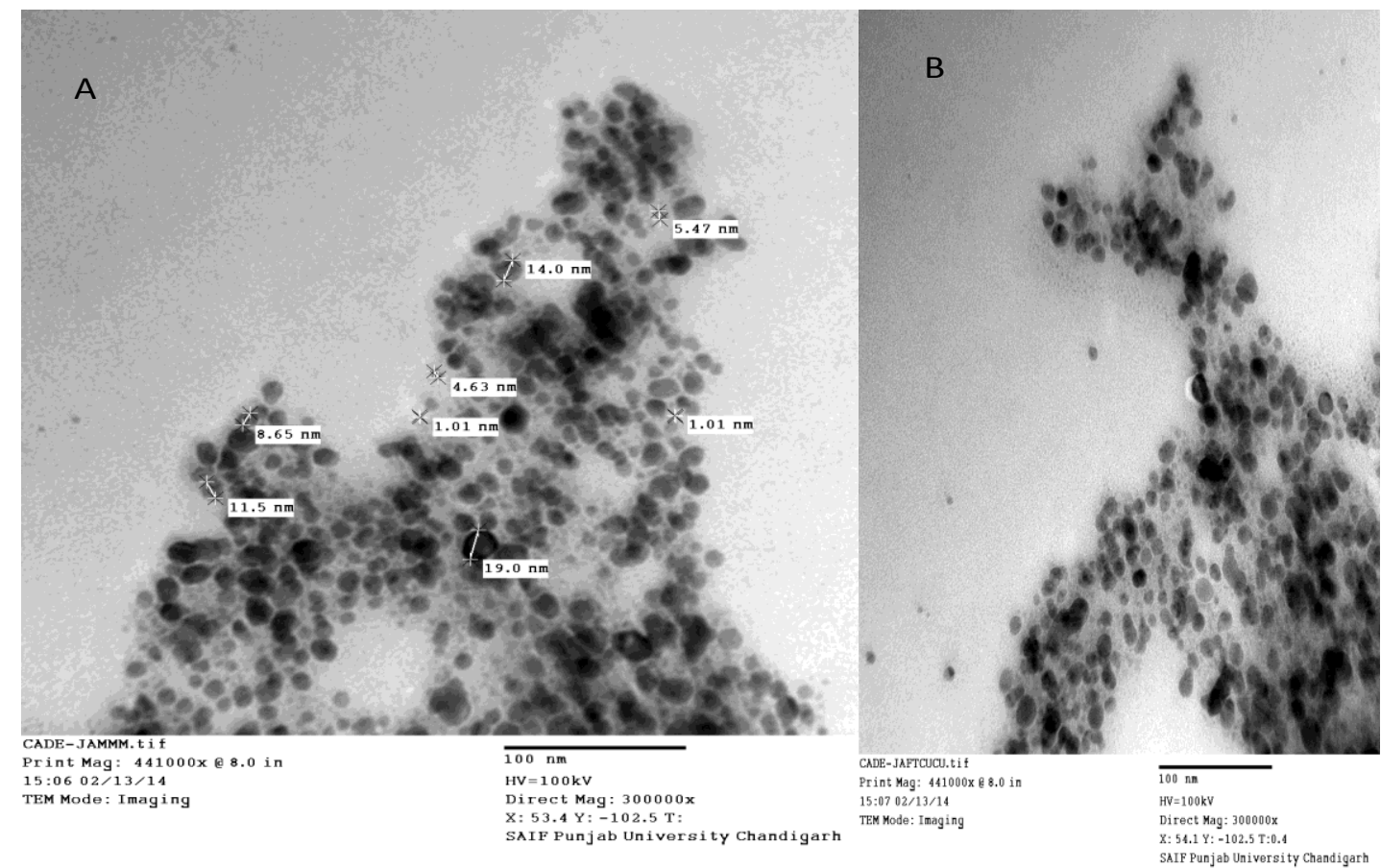

Fig.3 TEM images of silver nanoparticles at different magnification levels

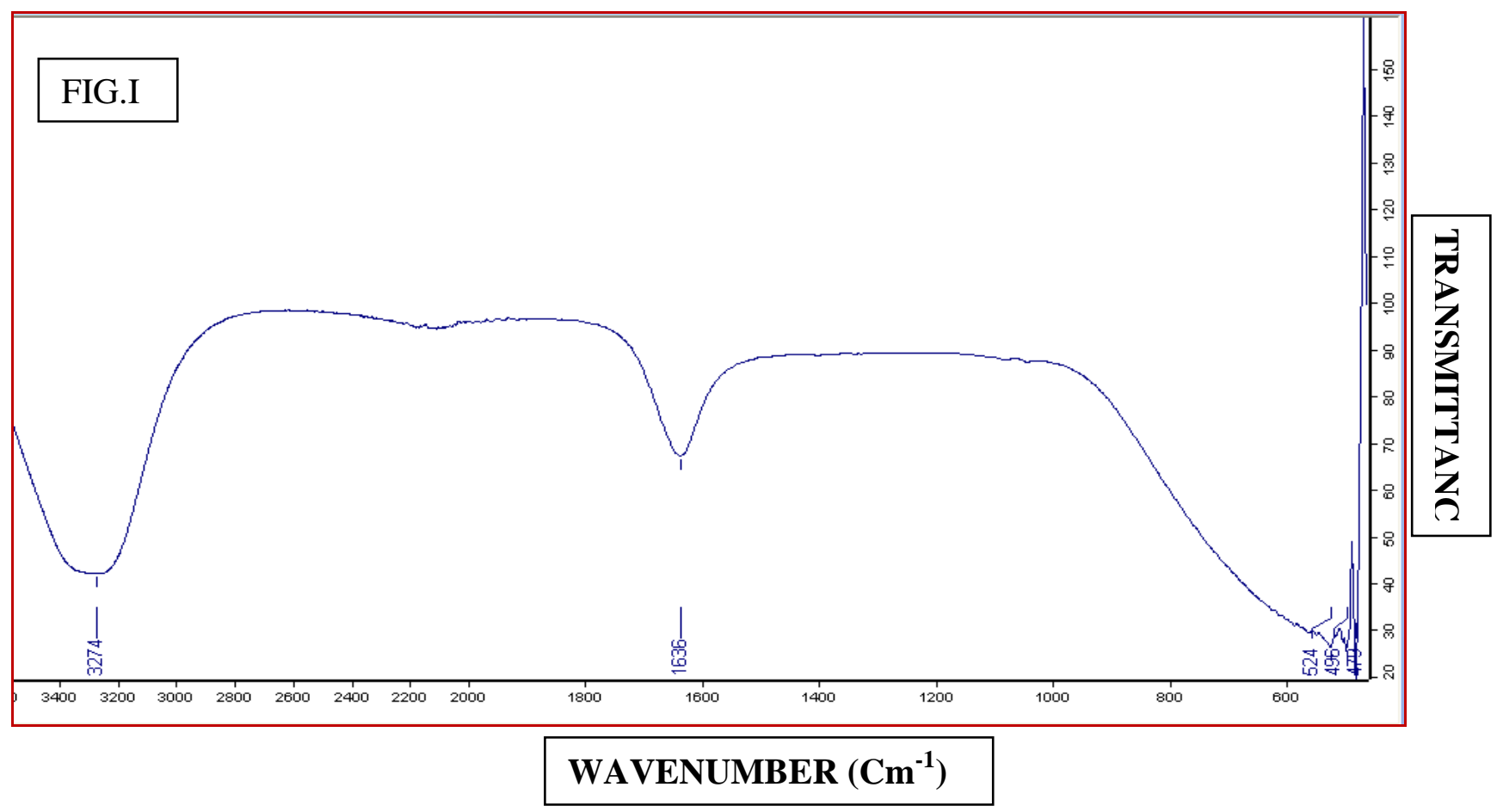

Fig.4 FTIR spectra of stem extract of $C$. decidua 


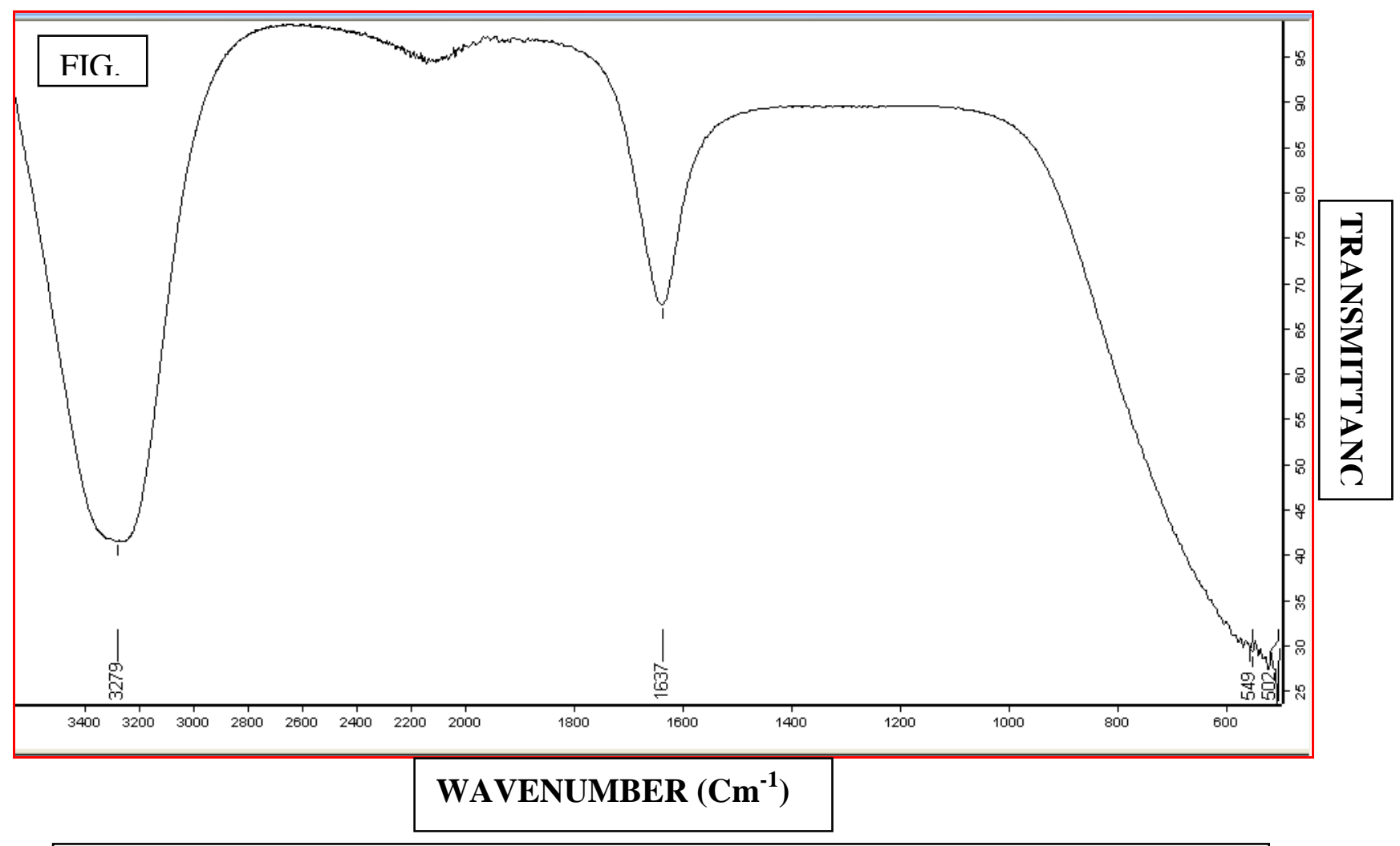

Fig.5 FTIR spectra of stem extract synthesized silver nanoparticles of C. decidua

\section{FTIR analysis}

FTIR absorption spectra of water dissolved Stem extract and the stem extract synthesized AgNPs (Figs. 4 and 5). FTIR can be used to analyze samples up to $\sim 11$ millimetres in diameter and either measure in bulk or the top $\sim 1$ micrometer layer. FTIR measurements were carried out to identify the possible biomolecules responsible for capping and efficient stabilization of the metal nanoparticles synthesized by Capparis decidua stem extract. The absorbance bands analysis in bioreduction are observed in the region of $470-4000 \mathrm{~cm}$ are 3274,1636 524,496 and $470 \mathrm{~cm}$. The peak observed at 3274 represents $\mathrm{N}-\mathrm{H}$ stretch (amines and amides) as the range of $3400-3250 \mathrm{~cm}^{-1}$ is amide region which is further associated with terminal Alkynes considered generally as stronger and sharper. The results are relevant to the earlier literature where binding as well as stabilization occurs by free amide groups present in proteinaceous substance used for synthesis (Rajesh et al., 2009; Sermakkani et al., 2012). The peak at found at 1636 i.e. between the range of $1614-1640$ is $\mathrm{C}-\mathrm{C}$ in alkene rings and $\mathrm{C}=\mathrm{C}$ stretch of aromatic rings, $524 \mathrm{~cm}^{-1}$ lies in the range of $690-515$ $\mathrm{cm}^{-1}$ is $\mathrm{C}-\mathrm{Br}$ stretch which is characteristic of alkyl halides. Whereas 470-496 $\mathrm{cm}^{-1}$ range may also be assigned to the first overtone mode of the methyl torsion vibration (Marimuthu et al., 2011) From the above observation it is assumed that the stabilisation is achieved by the identified compounds present in the plant extract. FTIR analysis was used to detect the characteristic peaks and their functional groups. The broadening of peaks indicated that the particles are polydispersed. The capping was confirmed by the existence of bands at 3279, 1637, 549 and $502 \mathrm{~cm}^{-1}$ (Ahlawat et al., 2015, Jyoti et al., 2016). 
Here we report a simplistic 'green' route for the synthesis of AgNPs using aqueous stem extract of Capparis decidua, and the characterization of the nanoparticles via UVVis spectroscopy, TEM analysis. Native Synthesized nanoparticles were showing UVVis absorption spectra at $460 \mathrm{~nm}$ with 0.94 . The TEM analysis indicates the presence of 1$20 \mathrm{~nm}$ size of silver Nanoparticles, which strongly support it to become a better microcidal agent. The synthesized silver nanoparticles were spherical in shape. The present study is eco-friendly, need cheaper cultivation requirements, higher growth rates on laboratory scales and use of biological agent in the synthesis which enhances lab to land approach. The stabilization of the nanoparticle is believed to occur by the presence of phytochemicals present in the plant extract and in recent years, the progress of efficient green chemistry approaches for the fabrication of commercially viable noble metallic nanoparticles has become a major focus of researchers. Moreover, this process could be easily scaled up for the industrial applications to increase the yield of the nanopartilces significantly, which undoubtedly would establish its commercial viability in medicine.

\section{Acknowledgement}

The authors would like to thank Department of Botany, M.D. University, Rohtak for providing facilities for carrying out this research work. We are grateful to SAIF (Sophisticated Analytical Instrumentation Facility) Punjab University Chandigarh for providing the TEM facilities. We also thankful to the Department of Genetics, M.D. University, Rohtak for providing FTIR facility.

\section{Conflict of Interest}

The Authors have no conflict of interest.

\section{References}

Gowramma, B., Keerthi, U., Mokula Rafi, Muralidhara Rao, D., 2015. Biogenic silver nanoparticles production and characterization from native stain of Corynebacterium species and its antimicrobial activity. 3 Biotech (2015) 5:195-201.

Harekrishna Bar, Dipak Kr Bhui, Gobinda P Sahoo, Priyanka Sarkar, Santanu Pyne, et al., 2009. Green synthesis of silver nanoparticles using seed extract of Jatropha curcas. Colloids and surfaces A: physicochem Eng Aspects 348: 212216.

Huang, H., and Yang, X., 2004. Synthesis of Polysaccharide-Stabilized Gold and Silver Nanoparticles: A Green Method. Carbohydrate Research. 339: 26272631.

Jalaluddin M. Ashraf, Mohammad Azam Ansari, Haris M. Khan, Mohammad A. Alzohairy, Inho Choi 2016. Green synthesis of silver nanoparticles and characterization of their inhibitory effects on AGEs formation using biophysical techniques. Scientific Reports 6:20414. DOI: 10.1038/srep20414.

Jegadeeswaran, P., Shivaraj, R., and Venckatesh, R. 2012. Green synthesis of silver nanoparticles from extract of Padina tetrastromatica leaf. Digest $J$ Nanomat. Biostruct. 7(3): 991-998.

Joyita banerjee, narendhirakannan, RT. 2011. Biosynthesis of silver nanoparticles from Syzygium cumini (1.) Seed extract and evaluation of their in vitro antioxidant activities. Digest Journal of nanomaterials and Biostructures 6(3): 961-968.

Jyoti Ahlawat, Anita R Sehrawat, Biological synthesis of silver nanoparticles using aqueous leaf extract of Capparis decidua (FORSK.) EDGEW: A better 
alternative. Journal of Pharmacy Research 2015, 9(4), 244-249.

Kreibig, U., and Vollmer, M. 1995. Optical properties of metal clusters. Springer series in material science. 25. SpringerVerlag, New York, pp 32-35.

Kumari Jyoti, Mamta Baunthiyal, Ajeet Singh, Characterization of silver nanoparticles synthesized using Urtica dioica Linn. leaves and their synergistic effects with antibiotics. Journal of Radiation Research and Applied Sciences 9 2016. 217 -227.

Li, S., Shen, Y., Xie, A., Yu, X., Qiu, L., Zhang, L., and Zhang, Q. 2007. Green synthesis of silver nanoparticles using Capsicum annuum L. extract. Green Chem. 9: 852-858 doi:10.1039/B615357G.

Mane Gavade, S.J., Nikam, G.H., Dhabbe, R.S., Sabale, S.R., Tamhankar, B.V., and Mulik, G.N. 2015. Green synthesis of silver nanoparticles by using carambola fruit extract and their antibacterial activity. Advances in Natural Sciences: Nanoscience and Nanotechnology. 6 (4): 1-6.

Marimuthu, S., Rahuman, A.A., Rajakumar, G. "Evaluation of green synthesized silver nanoparticles against parasites," Parasitology Research, vol. 108, no. 6, pp. 1541-1549, 2011.

Medda, S., Hajra, A., Dey, U., Bose, P., and Mondal, N.K. 2014. Biosynthesis of silver nanoparticles from Aloe vera leaf extract and antifungal activity against Rhizopus sp. and Aspergillus sp. Applied Nanoscience: 1-16.

Mittal, J., Batra, A., Singh, A., and Sharma, M. M. 2014. Adv. Nat. Sci: Nanosci. Nanotechnol. 5043002.

Mulvaney, P., 1996. Surface plasmon spectroscopy of nanosized metal particles. Langmuir. 12: 788- 800.

Philip, D., 2010. Rapid Green Synthesis of Spherical Gold Nanoparticles Using
Mangifera indica Leaf. Spectrochimica Acta Part A: Molecular and Biomolecular Spectroscopy. 77: 807810.

Rathee, S., Mogla, O.P., Rathee, P., and Rathee, D. 2010. Quantification of $\beta$ Sitosterol using HPTLC from Capparis decidua. Der Pharma Chemica. 2 (4): 86-92.

Sai Lakshmi, G., Johnson, M., Revathy, I., and Shibila, T. 2015. Green synthesis of silver nanoparticles and its characterization in Lobophora variegate J. V. Lamouroux. In: Proceeding of Conservation and Suitable Utilization of Marine Resources. Pp. 90-93. 2.

Saifuddin, N., Wong, C.W., and Nur yasumira, A. A. 2009. Rapid biosynthesis of silver nanoparticles using culture supernatant of bacteria with microwave irradiation. E-J Chem 6:61-70.

Sermakkani, M., and V, Thangapandian. 2012. Gc-Ms Analysis of Cassia Italica Leaf Methanol Extract. Asian Journal of Pharmaceutical And Clinical Research. 5 (2): 90-94 Issn - 0974-2441.

Shakeel Ahmed, Saifullah, Mudasir Ahmad, Babu Lal Swami, Saiqa Ikram. Green synthesis of silver nanoparticles using Azadirachta indica aqueous leaf extract. Journal of Radiation Research and Applied Science 9 2016. 1-7.

Sharma, V.K., Yngard, R.A. and Lin, Y. 2009. Silver Nanoparticles: Green Synthesis and Their Antimicrobial Activities. Advances in Colloid and Interface Science. 145: 83-96.

Shibila, T., and Johnson, M. 2015. Green synthesis of silver nanoparticles and evaluate the cytotoxic potentials of Laurencia pedicularioides Borgesen. In: Proceeding of Conservation and Suitable Utilization of Marine Resources. Pp. 74-77. 
Shin, H. S., Yang, H. J., Kim, S. B., and Lee, M. S. 2004. Mechanism of growth of colloidal silver nanoparticles stabilized by polyvinyl pyrrolidone in gammairradiated silver nitrate solution. $J$. Colloid Interf. Sci. 274: 89.

Song, J.Y., and Kim, B.S. 2009. Rapid Biological Synthesis of Silver Nanoparticles Using Plant Leaf Extracts. Bioprocess and Biosystems Engineering. 32: 79-84.

Sun, Y. P., Atorngitjawat, P., and Meziani, M.

J. 2001. Preparation of silver nanoparticles via rapid expansion of water in carbon dioxide microemulsion into reductant solution. Langmuir 17:5707-5710.

Sundeep Dola, Vijaya Kumar, T., Subba Rao, P.S., Ravikumar, R. V. S. S. N., Gopala, A, Krishna. (2017) Green synthesis and characterization of $\mathrm{Ag}$ nanoparticles from Mangifera indica leaves for dental restoration and antibacterial applications. Pro Biomater (2017) 6:5766.

\section{How to cite this article:}

Jyoti Ahlawat and Anita R. Sehrawat. 2017. Nano Dimensional (1-20nm) Silver Nanoparticles: Stem Extract of Capparis decidua (FORSK.) EDGEW Mediated Synthesis and its Characterization-A Lab to Land Approach. Int.J.Curr.Microbiol.App.Sci. 6(10): 1874-1883. doi: https://doi.org/10.20546/ijcmas.2017.610.226 